\title{
BMJ Open Is palliative care support associated with better quality end-of-life care indicators for patients with advanced cancer? A retrospective cohort study
}

\author{
Lucy E Ziegler, ${ }^{1}$ Cheryl L Craigs, ${ }^{1}$ Robert M West, ${ }^{2}$ Paul Carder, ${ }^{3}$ Adam Hurlow, ${ }^{4,5}$ \\ Pablo Millares-Martin, ${ }^{6}$ Geoff Hall, ${ }^{4}$ Michael I Bennett ${ }^{1}$
}

To cite: Ziegler LE, Craigs CL, West RM, et al. Is palliative care support associated with better quality endof-life care indicators for patients with advanced cancer? A retrospective cohort study. BMJ Open 2018;8:e018284. doi:10.1136/ bmjopen-2017-018284

- Prepublication history and additional material for this paper are available online. To view these files, please visit the journal online (http://dx.doi. org/10.1136/bmjopen-2017018284).

Received 19 June 2017 Revised 11 December 2017 Accepted 15 December 2017

Check for updates

${ }^{1}$ Academic Unit of Palliative Care, Leeds Institute of Health Sciences, University of Leeds, Leeds, UK

${ }^{2}$ Health Services Research, Leeds Institute of Health

Sciences, University of Leeds, Leeds, UK

${ }^{3} \mathrm{NHS}$ Bradford Districts Clinical Commissioning Group, Bradford, UK

${ }^{4}$ Leeds General Infirmary, Leeds, UK

${ }^{5}$ Specialist Palliative Care Unit, Leeds Teaching Hospitals NHS

Trust, Leeds, UK

${ }^{6}$ Leeds West Clinical

Commissioning Group, Leeds, UK

Correspondence to

Dr Lucy E Ziegler;

l.e.ziegler@leeds.ac.uk

\section{ABSTRACT}

Objectives This study aimed to establish the association between timing and provision of palliative care (PC) and quality of end-of-life care indicators in a population of patients dying of cancer.

Setting This study uses linked cancer patient data from the National Cancer Registry, the electronic medical record system used in primary care (Systm0ne) and the electronic medical record system used within a specialist regional cancer centre. The population resided in a single city in Northern England.

Participants Retrospective data from 2479 adult cancer decedents who died between January 2010 and February 2012 were registered with a primary care provider using the Systm0ne electronic health record system, and cancer was certified as a cause of death, were included in the study.

Results Linkage yielded data on 2479 cancer decedents, with $64.5 \%$ who received at least one PC event. Decedents who received $\mathrm{PC}$ were significantly more likely to die in a hospice $(39.4 \%$ vs $14.5 \%, \mathrm{P}<0.005)$ and less likely to die in hospital $(23.3 \%$ vs $40.1 \%, P<0.05)$, and were more likely to receive an opioid ( $53 \%$ vs $25.2 \%, P<0.001)$. PC initiated more than 2 weeks before death was associated with avoiding a hospital death ( $\geq 2$ weeks, $P<0.001$ ), more than 4 weeks before death was associated with avoiding emergency hospital admissions and increased access to an opioid ( $\geq 4$ weeks, $P<0.001$ ), and more than 33 weeks before death was associated with avoiding late chemotherapy ( $\geq 33$ weeks, no chemotherapy $P=0.019$, chemotherapy over 4 weeks $\mathrm{P}=0.007$ ).

Conclusion For decedents with advanced cancer, access to $\mathrm{PC}$ and longer duration of PC were significantly associated with better end-of-life quality indicators.

\section{INTRODUCTION}

Integration of palliative care alongside oncology management should be considered early in the course of illness for patients with metastatic cancer or high symptom burden, according to American Society of Clinical Oncology guidelines. ${ }^{1}$ This recommendation is based on a number of randomised controlled trials, largely from North America,
Strengths and limitations of this study

- To the best of our knowledge, this is the first UK study to explore the associations between duration of palliative care and quality of end-of-life care in a large population of cancer decedents.

- The data used in this study are derived from a live clinical system and as such are likely to represent errors or omissions inherent within that system.

- The definition of good quality end-of-life care used in this study is informed by UK policy and guidance on end-of-life care provision. We recognise that what constitutes good quality care at the end of life can vary by individual and that the study does not capture individual preferences or circumstances.

which found early palliative care was associated with improved quality of life and a reduction in acute hospital admissions and aggressive cancer treatments at the end of life. ${ }^{2-6}$ Though there were inconsistencies across trials, in general common characteristics were an assessment and several follow-up consultations by specialist palliative care teams over a period of 2-3 months, which occurred about 6-14 months before patients died.

Compared with patients recruited to these clinical trials, patients with cancer in routine care are often referred to palliative care services much later in the course of their illness. ${ }^{7}$ We recently showed that for 4650 patients with cancer in Leeds, median contact was 34 days for community and hospital palliative care services. ${ }^{8}$ The relatively short duration of palliative care in routine services might limit the opportunity for identification of needs and the subsequent provision of effective support and symptom management. This could adversely impact on end-of-life outcomes. 
Systematic reviews and pooled analyses of routinely collected data have demonstrated an association between palliative care intervention and increased proportion of home deaths as well as reduction in emergency admissions. ${ }^{9} 10$ However, no study has quantified these associations in relation to duration of palliative care. In order to more directly inform models of service delivery, better quality data are needed on how long patients with cancer need to receive palliative care before important improvements in end-of-life care can be observed.

We report a retrospective cohort study that linked routinely collected data on hospital and community healthcare resource use in cancer decedents.

We chose this study design because it enabled us to examine the effects of palliative care service delivery in routine care for a case series of cancer decedents and minimised recruitment bias from a clinical trial design. We wanted to test the hypothesis that contact with and longer duration of palliative care would be associated with better end-of-life care quality indicators for patients with advanced cancer.

\section{METHODS}

\section{Study population}

Retrospective data from 2479 adult (aged at least 18 years at death) cancer decedents who died between January 2010 and February 2012, resided within a single UK city, were registered with a primary care provider using the SystmOne electronic health record system, and cancer was certified as a cause of death, were included in the study.

\section{Data collection}

Data were obtained from three sources: the Northern and Yorkshire Cancer Registry and Information Service (NYCRIS), SystmOne and the Patient Pathway Manager (PPM). NYCRIS maintains a database of all cancers occurring in the Northern and Yorkshire region in England. SystemOne is an electronic health record system used by approximately $75 \%$ of primary care providers in Leeds. PPM is a clinical information system used at a regional specialist cancer centre to manage and coordinate patient care.

Decedents eligible for the study were identified from the NYCRIS database based on address, date of death and cause of death. The NYCRIS database provided all demographic, diagnostic and death information. SystmOne provided opioid prescription information and community palliative care provision. PPM provided chemotherapy treatment, emergency hospital admissions and hospital-based palliative care referral information. The three data sources were linked using an open pseudonymiser system to create an encrypted code based on National Health Service numbers.

\section{Assessment of palliative care provision}

The primary measure of palliative care provision used in this study was time between first contact with palliative care and death, measured as time in weeks. Both hospital-based and community-based records of palliative care events were included.

The PPM system provided information on hospital-based palliative care referrals. For each patient included in our study, every unique palliative care referral date recorded on the PPM system was identified as a unique palliative care event. Community palliative care provision was estimated using general practitioner (GP) communications within SystmOne, based on a multistage approach. In the first stage, any GP communication within SystmOne which included either a palliative care-based READ code or included text indicating palliative care, based on a keyword search, was extracted from SystemOne as a list or records, with the possibility of multiple records per patient. In the second stage, only records which extended up to the date of death and included either a READ code indicating the active provision of palliative care, identified through consensus between authors (see online supplementary appendix 1) or included communication with a hospice, were identified as representing palliative care provision. For each patient, every unique palliative care provision date recorded in SystmOne was identified as a unique palliative care event representing contact with a palliative care team member.

The total number of palliative care events identified for each patient was a secondary measure of palliative care provision which was used as a proxy to indicate the extent of palliative care support provided.

\section{Outcomes}

The end-of-life quality indicators assessed were informed by UK policy and international research evidence on what constitutes quality end-of-life care provision for patients with cancer $^{3511-15}$ and included place of death, access to strong opioids within the last year of life (identified if decedents received at least one opioid prescription within the last 12 months of life), timing of last chemotherapy treatment and emergency hospital admission up to 4 weeks before death. We chose these because a reduction in hospital use at the end of life is commonly used as a proxy for better quality care. ${ }^{3511-15}$ Recently, we have demonstrated the relatively late onset of strong opioid prescribing before death in a cohort of patients with cancer. ${ }^{16}$ We judged that given the prevalence of pain in advanced cancer access to strong opioids could also be a proxy for better quality care.

Place of death was categorised as own home, hospice, hospital, care home, other or unknown. Decedents with at least one strong opioid prescription were coded as yes, with prescriptions other than a strong opioid within the last year were coded as no. The list of strong opioids included are provided in online supplementary appendix 2. Decedents without a prescription were coded as missing. The timing of chemotherapy was categorised into either 
no chemotherapy, chemotherapy $0-4$ weeks before death or chemotherapy over 4 weeks before death. The number of emergency admissions to hospital in last 4 weeks of life were grouped into avoided emergency admission or did not avoid emergency admission (one or more emergency admissions).

\section{Covariates}

Covariates considered were age, categorised into younger than 50 years, older than 80 years and deciles in between: sex (male or female); Indices of Multiple Deprivation (IMD) quintile (where $1=$ most deprived and $5=$ least deprived); cancer diagnosis and duration of illness (in years) before death.

\section{Statistical analysis}

We used Pearson's $\chi^{2}$ to test associations between receiving palliative care and end-of-life quality outcomes. Post hoc $\chi^{2}$ tests were conducted for each possible 2 by 2 table comparison, adjusted using the Bonferroni correction, where outcomes included more than two categories and the $\chi^{2}$ resulted in a $P$ value of less than 0.05 . The association between duration of palliative care and number of palliative care events was explored through frequency tables and the Spearman's rank correlation coefficient. Differences in median scores were compared using the Mann-Whitney U test, for two group comparisons, or the Kruskal-Wallis $\mathrm{H}$ test, for more than two groups. Where statistically significant results were identified from the Kruskal-Wallis H test, Dunn-Bonferroni post hoc tests for multiple comparisons of rank sums, based on the z-statistics, were conducted for each possible combination of two group comparisons. ${ }^{17}$

We constructed a classification tree for each end-of-life care outcome, using $\chi^{2}$ automatic interaction detection (CHAID), to identify, for each outcome, the optimum cut-off points for duration of palliative care. ${ }^{18}$ Each CHAID classification tree included palliative care duration as the only predictor variable. Nodes associated with the first branch of the dendrogram identified the optimum duration of palliative care cut-off points. Where the cut-off point was 0 weeks palliative care, this reflected contact with palliative care but within 7 days of death. Multivariable regression models (logistic and multinomial) were used to investigate the role of these cut-off points on end-of-life care outcomes, after controlling for age at death, gender, IMD deprivation quintile, first diagnosis cancer site and duration of illness. Results are presented as ORs alongside $95 \%$ CIs.

Complete case analysis was undertaken. $\mathrm{P}$ values less than 0.05 were considered statistically significant (two-tailed). Analysis was conducted using IBM SPSS statistics V.23.

\section{Public involvement}

Patients were involved in setting the research question and in the design of the study, no patients were asked for advice on interpretation or writing up of results. The results of the research have been disseminated to the patient community through patient forums.

\section{RESULTS}

\section{Patient characteristics}

Of the 2479 patients included in the study $64.5 \%$ ( $n=1598)$ received at least one palliative care event. Community-based palliative care was received by $45.3 \% \quad(n=1124)$, and hospital-based palliative care was received by $40.0 \%$ $(\mathrm{n}=991)$, of patients.

Palliative care was more likely to be received by decedents who were younger $(\mathrm{P}<0.001)$ or female $(\mathrm{P}=0.006)$. Cancer diagnosis $(\mathrm{P}=0.004)$ was significantly associated with receiving palliative care. Decedents with upper gastrointestinal cancers were significantly more likely to receive palliative care $(\mathrm{P}<0.05)$ while decedents with lung cancer $(\mathrm{P}<0.05)$ or cancers of the central nervous system $(\mathrm{P}<0.05)$ were significantly less likely to receive palliative care (table 1).

The time between first contact with palliative care and death varied widely from less than 1-343 weeks, with a median interval of 6 weeks (IQR 2-19 weeks). Most decedents who received palliative care received between one and three palliative care events (median 2 events, IQR 1-3 events). There was a significant positive relationship between the interval from first contact to death and number of palliative care events $\left(r_{s}=0.535, \mathrm{P}<0.001\right)$.

\section{Place of death}

Place of death was significantly associated with palliative care provision $(\mathrm{P}<0.001)$. Post hoc tests showed that patients who received palliative care were significantly more likely to die in a hospice $(39.4 \%$ vs $14.5 \%, \mathrm{P}<0.05)$ and significantly less likely to die in hospital (23.3\% vs $40.1 \%, \mathrm{P}<0.05)$, at home $(26.8 \%$ vs $31.8 \%, \mathrm{P}<0.05)$ or in a care home $(8.7 \%$ vs $12.0 \%, \mathrm{P}<0.05)$ compared with patients who did not receive palliative care (table 2).

For the 1598 decedents who received palliative care, a shorter time between first contact with palliative care and death was observed for hospital deaths (median 3 weeks palliative care) compared with deaths in hospice (median 7 weeks palliative cares), at home (median 7 weeks palliative care) or in a care home (median 13 weeks palliative care) $(\mathrm{P}<0.001)$. There was also a significant difference in the number of palliative care events by place of death $(\mathrm{P}<0.001)$, with the median number of palliative events in hospital equalling one event, compared with a median of two events for deaths at home, in a hospice or in a care home (table 2).

\section{Receiving at least one strong opioid prescription within the last year of life}

Decedents who received palliative care were significantly more likely to have also been prescribed strong opioids before death compared with patients who did not receive palliative care $(53.9 \%$ vs $25.2 \%, \mathrm{P}<0.001)$. 
Table 1 Characteristics of the study population by palliative care provision

\section{Characteristics}

Palliative care provision $(\mathrm{n}=\mathbf{2 4 7 9})$

Total no of patients: No row \%

Age at death (years): No column \%

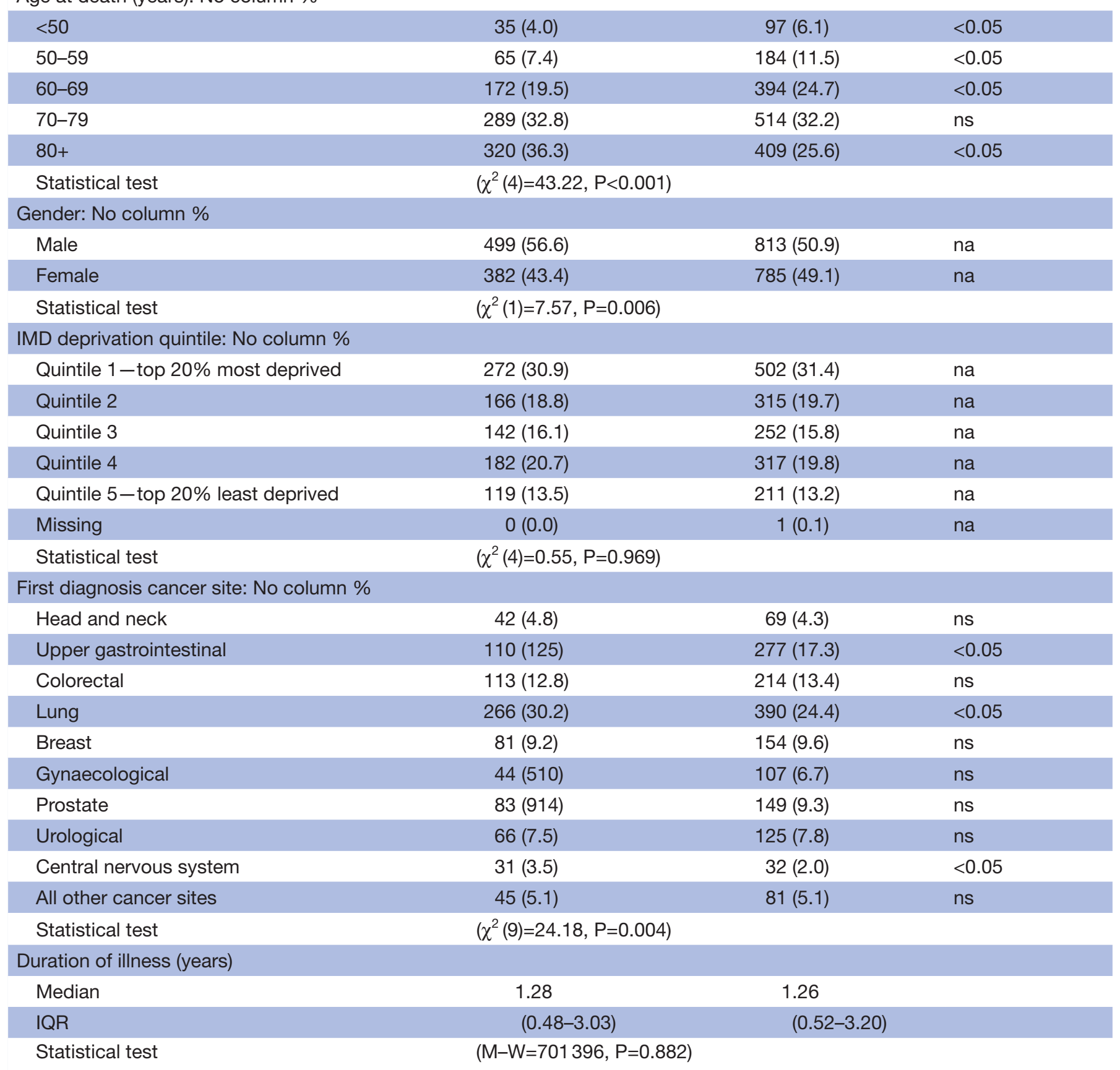

\section{$\chi^{2}$, (df shown in brackets).}

IMD, Indices of Multiple Deprivation; M-W, Mann-Whitney U test; na, not applicable; ns, not significant.

For those decedents who received palliative care $(\mathrm{n}=1598)$, the the time between first contact with palliative care and death and the number of palliative care events were significantly higher for decedents who received at least one strong opioid prescription (median 9 weeks palliative care vs 4 weeks palliative care, $\mathrm{P}<0.001$; median 2 palliative care events vs 1 palliative care event, $\mathrm{P}<0.001$ ) (table 3 ).

\section{Palliative care provision $(n=2479)$}

Not received

Received

Post hoc

1598 (64.5)

Timing of last chemotherapy

A significant relationship was identified between timing of last chemotherapy and receiving palliative care $(\mathrm{P}<0.001)$. Post hoc analysis showed that those who received palliative care were more likely to have been treated with chemotherapy at any point during the course of their disease $(63.6 \%$ vs $47.4 \%, \mathrm{P}<0.05)$ and were more 
Table 2 Palliative care provision by place of death

Palliative care provision $(n=2479)$

Subgroup receiving palliative care $(n=1598)$

Time between first contact with palliative care and

\begin{tabular}{|c|c|c|c|c|c|c|c|}
\hline \multirow[b]{2}{*}{ Place of death } & \multirow{2}{*}{$\begin{array}{l}\text { Not received } \\
\text { No }(\%)\end{array}$} & \multirow{2}{*}{$\begin{array}{l}\text { Received } \\
\text { No (\%) }\end{array}$} & \multirow[b]{2}{*}{ Post hoc } & \multicolumn{2}{|c|}{ No of palliative events } & \multicolumn{2}{|c|}{ death } \\
\hline & & & & Median (IQR) & Post hoc & Median (IQR) & Post hoc \\
\hline Own home & $280(31.8)$ & $429(26.8)$ & $<0.05$ & 2 events (1-3) & i & 7 weeks $(2.5-17)$ & i, ii \\
\hline Hospital & $353(40.1)$ & $372(23.3)$ & $<0.05$ & 2 events (1-2) & i, ii, iii & 3 weeks (1-14) & i, iii, v \\
\hline Care home & $106(12.0)$ & $139(8.7)$ & $<0.05$ & 2 events (1-3) & iii & 13 weeks (4-35) & ii, iv, v \\
\hline Unknown & $13(1.5)$ & $29(1.8)$ & - & - & & - & \\
\hline
\end{tabular}

Statistical test $\quad\left(\chi^{2}(3)=180.52, P<0.001\right)$

$(\mathrm{K}-\mathrm{W}(3)=128.14, \mathrm{P}<0.001)$

$(\mathrm{K}-\mathrm{W}(3)=75.77, \mathrm{P}<0.001)$

$\chi^{2}$, (df shown in brackets); Post hoc, multiple comparison z-test statistics comparing mean ranks for each possible two category comparison group; i, ii, iii, iv, v links categories where post hoc comparison groups which resulted in a $\mathrm{P}$ value less than 0.05 (after adjusting using the Bonferroni correction).

$\mathrm{K}-\mathrm{W}$, Kruskal-Wallis $\mathrm{H}$ test.

likely to have stopped chemotherapy over 4 weeks before death, compared with those not receiving palliative care ( $58.5 \%$ vs $42.1 \%, \mathrm{P}<0.05)$.

For patients who received palliative care $(n=1598)$ the time between first contact with palliative care and death was significantly associated with the timing of latest chemotherapy $(\mathrm{P}<0.001)$ (table 4$)$.

\section{Emergency hospital admission within the last 4 weeks of life}

The majority of the sample (1926 out of $2479,77.7 \%$ ) avoided emergency hospital admission in the last 4 weeks of life. A borderline significant association was identified between emergency hospital admissions in the last 4 weeks of life and receiving palliative care $(\mathrm{P}=0.049)$. For decedents who received palliative care $(n=1598)$, emergency admission was associated with a significantly shorter time between first contact with palliative care and death ( 4 weeks vs 7 weeks, $\mathrm{P}<0.001$ ) and significantly fewer palliative care events overall (2 events vs 2 events, $\mathrm{P}=0.010$ ) (table 5).

\section{Multivariable regression}

Classification tree optimum cut-off points for each of the end-of-life outcomes are provided in online supplementary appendix 3. Between three and five optimum cut-off points were identified for the four end-of-life outcomes in relation to the time between first contact with palliative care and death. The multivariable (multinomial) logistic regression models, showed overall greater ORs for better outcomes at the end of life with longer time between first contact with palliative care and death figure 1. Each cut-off point within the model generally represents a significantly better outcome. For example, although overall there was no association between palliative care and increased home deaths, the model shows that decedents who received 2-7 weeks of palliative care had 2.96 better odds of dying at home than in hospital (95\% CI 2.02 to $4.35, \mathrm{P}<0.001)$, and those who received more than 8 weeks of palliative care had 3.49 better odds of dying at home (95\% CI 2.42 to 5.04 , $\mathrm{P}<0.001)$. Similarly, there was a clear stepwise increase in the odds of receiving an opioid prescription with longer time between first contact with palliative care and death (Not received palliative care: OR $0.49,95 \%$ CI 0.39 to $0.61, \mathrm{P}<0.001 ; 0-3$ weeks palliative care: OR $1.00,95 \%$ $\mathrm{CI}=$ reference; $4-7$ weeks palliative care: $\mathrm{OR} 1.49,95 \%$ CI 1.12 to $1.98, \mathrm{P}=0.006 ; 8-32$ weeks palliative care:

Table 3 Palliative care provision by strong opioid prescription within the last twelve months of life

\section{Palliative care provision $(n=2479)$ Subgroup receiving palliative care $(n=1598)$}

\begin{tabular}{|c|c|c|c|c|}
\hline \multirow{2}{*}{$\begin{array}{l}\text { Strong opioid } \\
\text { prescription within last } \\
\text { year of life }\end{array}$} & \multirow{2}{*}{$\begin{array}{l}\text { Not received } \\
\text { No }(\%)\end{array}$} & \multirow{2}{*}{$\begin{array}{l}\text { Received } \\
\text { No (\%) }\end{array}$} & \multirow{2}{*}{$\begin{array}{l}\text { No of palliative events } \\
\text { Median (IQR) }\end{array}$} & \multirow{2}{*}{$\begin{array}{l}\text { Time between first contact } \\
\text { with palliative care and death } \\
\text { Median (IQR) }\end{array}$} \\
\hline & & & & \\
\hline No & 655 (74.3) & $736(46.1)$ & 1 event (1-2) & 4 weeks (1-12) \\
\hline Missing & $4(0.5)$ & $0(0.0)$ & & \\
\hline Statistical test & \multicolumn{2}{|c|}{$\left(\chi^{2}(1)=188.54, P<0.001\right)$} & $(\mathrm{M}-\mathrm{W}=226447, \mathrm{P}<0.001)$ & $(\mathrm{M}-\mathrm{W}=233259, \mathrm{P}<0.001)$ \\
\hline
\end{tabular}

$\chi^{2}$, (df shown in brackets).

M-W, Mann-Whitney U test. 
Table 4 Palliative care provision by timing of latest chemotherapy

Palliative care provision $(n=2479)$

Subgroup receiving palliative care $(n=1598)$

Time between first contact with palliative care and

\begin{tabular}{|c|c|c|c|c|c|c|c|}
\hline \multirow{2}{*}{$\begin{array}{l}\text { Time of latest } \\
\text { chemotherapy }\end{array}$} & \multirow{2}{*}{$\frac{\text { Not received }}{\text { No }(\%)}$} & \multirow{2}{*}{$\begin{array}{l}\text { Received } \\
\text { No }(\%)\end{array}$} & \multirow[b]{2}{*}{ Post hoc } & \multicolumn{2}{|c|}{ No of palliative events } & \multicolumn{2}{|c|}{ death } \\
\hline & & & & Median (IQR) & Post hoc & Median (IQR) & Post hoc \\
\hline No chemotherapy & $463(52.6)$ & $582(36.4)$ & $<0.05$ & 2 events (1-3) & i & 5 weeks (2-14) & $\mathrm{i}, \mathrm{ii}$ \\
\hline Over 4 weeks & $371(42.1)$ & 934 (58.5) & $<0.05$ & 2 events (1-3) & ii & 8 weeks (2-22) & ii, iii \\
\hline Statistical test & \multicolumn{2}{|c|}{$\left(\chi^{2}(2)=63.90, P<0.001\right)$} & & \multicolumn{2}{|c|}{$(K-W(2)=19.94, P<0.001)$} & \multicolumn{2}{|c|}{$(K-W(2)=46.58, P<0.001)$} \\
\hline
\end{tabular}

$\chi^{2}$, (df shown in brackets). Post hoc, multiple comparison z-test statistics comparing mean ranks for each possible two category comparison group; i, ii, iii, iv, v links categories where post hoc comparison groups which resulted in a $\mathrm{P}$ value less than 0.05 (after adjusting using the Bonferroni correction).

$\mathrm{K}-\mathrm{W}$, Kruskal-Wallis $\mathrm{H}$ test; ns, not significant.

OR 2.45, 95\% CI 1.90 to $3.16, \mathrm{P}<0.001 ; 33+$ weeks palliative care: OR $3.24,95 \%$ CI 2.34 to $4.49, \mathrm{P}<0.001)$.

\section{DISCUSSION}

Our analysis confirms existing research that better outcomes at the end of life are associated with access to palliative care services. However, we have been able to demonstrate for the first time that longer interval between first contact with palliative care and death is associated with increasingly better outcomes, specifically relating to place of death outside hospital, access to strong opioid, ${ }^{19}$ and avoiding chemotherapy and emergency hospital admission within the last 4 weeks of life. For some outcomes such as place of death at home, there appears to be a minimum interval between first contact with palliative care and death that is associated with higher odds of home death. This suggests that sufficient time is required to plan and coordinate in order to achieve this outcome for a patient.

We found decedents who received palliative care were less likely to die in hospital and more likely to die in a hospice. While it is important to acknowledge that for some patients dying in hospital represents appropriate end-of-life care, for most, care is rated significantly lower for people who die in a hospital, compared with home, a hospice or care home. ${ }^{20}$ Despite this, approximately $48 \%$ of UK patients with cancer die in hospital. ${ }^{21}$ We identified the level of palliative care involvement associated with a reduction in hospital deaths was minimal (two contacts initiated at least 3 weeks before death). The potential per patient saving by avoiding a hospital death proposed by the National End of Life Information Network is $£ 958$ per patient. $^{22}$

Evidence for the impact of palliative care on home death is inconsistent. We found the rate of home deaths in decedents who received palliative care was lower compared with those who did not, however, the likelihood of dying at home, rather than hospital, increased as the level of palliative care involvement increased. A meta-analysis found palliative care had no impact on home deaths ${ }^{23}$ while a Cochrane review undertaken the same year found it more than doubles the odds of dying at home. ${ }^{24}$ These differences may reflect differences in the availability of hospice or palliative care services, or bias in the selection of suitable patients for palliative care. Our data suggest that the interval between first contact with palliative care and death may account for this inconsistent relationship.

Opioid analgesia is the recommended treatment for moderate to severe pain $^{25}$ the prevalence of which in advanced cancer is estimated to be between $62 \%$ and $86 \%{ }^{26}$ and patients who die of cancer typically require

Table 5 Palliative care provision by emergency hospital admissions within the last 4 weeks of life

\section{Palliative care provision $(n=2479)$}

Subgroup receiving palliative care $(n=1598)$

Avoided emergency hospital
admissions 0-4 weeks
before death
Yes

No (one or more admission)

Statistical test

\begin{tabular}{|c|c|}
\hline Not received & Received \\
\hline No (\%) & No (\%) \\
\hline 704 (79.9) & $1222(76.5)$ \\
\hline 177 (20.1) & 376 (23.5) \\
\hline
\end{tabular}

No of palliative events

Median (IQR) Median (IQR)

2 events $(1-3)$

7 weeks (2-20)

2 events (1-3)

4 weeks (1-12)

Time between first contact with palliative care and death

$\chi^{2}$, (df shown in brackets).

$\mathrm{M}-\mathrm{W}$, Mann-Whitney $\mathrm{U}$ test. 


\section{End of life outcomes}

Place of death (reference=Hospital) $p<0.001$

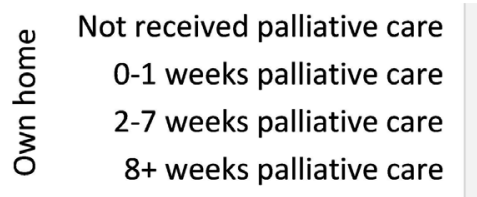

Not received palliative care

0-1 weeks palliative care

2-7 weeks palliative care

$8+$ weeks palliative care

Not received palliative care

0-1 weeks palliative care

2-7 weeks palliative care

$8+$ weeks palliative care
H

I

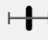

H

\section{Odds Ratio $(95 \% \mathrm{Cl}) \quad$ P value \\ 1.59 (1.15 to 2.22$) \quad p=0.006$ \\ 1.00 (reference) \\ $2.96(2.02$ to 4.35$) \quad p<0.001$ \\ $3.49(2.42$ to 5.04$) \quad p<0.001$}

H

1

H

$+$

$0.51(0.37$ to 0.70$) \quad p<0.001$

1.00 (reference)

$3.23(2.29$ to 4.57$) p<0.001$

$3.22(2.30$ to 4.50$) \quad p<0.001$

$3.34(1.73$ to 6.45$) p<0.001$

1.00 (reference)

$4.53(2.19$ to 9.39$) \quad p<0.001$

$9.81(4.96$ to 19.40$) \quad p<0.001$

\section{Opioid prescription within last year of life (reference $=$ No) $p<0.001$}

\begin{tabular}{|c|c|c|}
\hline Not received palliative care & & 0.49 (0.39 to 0.61$) \quad p<0.001$ \\
\hline $0-3$ weeks palliative care & 1 & 1.00 (reference) \\
\hline 4-7 weeks palliative care & H & $1.49(1.12$ to 1.98$) p=0.006$ \\
\hline 8-32 weeks palliative care & $\boldsymbol{H}$ & 2.45 (1.90 to 3.16$) \quad p<0.001$ \\
\hline $33+$ weeks palliative care & H & $3.24(2.34$ to 4.49$) p<0.06$ \\
\hline
\end{tabular}

Time of latest chemotherapy (reference $=0-4$ weeks before death) $p<0.001$
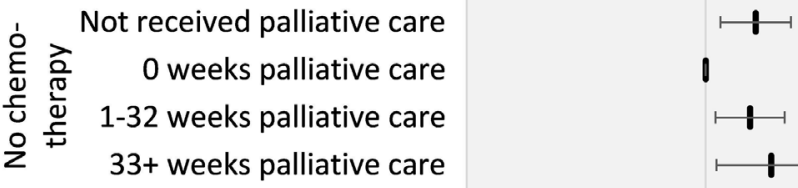

1
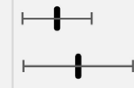

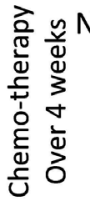

Not received palliative care

0 weeks palliative care

1-32 weeks palliative care

$33+$ weeks palliative care

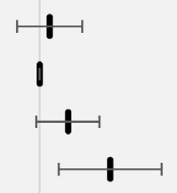

$2.62(1.33$ to 5.17$) \quad p=0.006$

1.00 (reference)

2.35 (1.21 to 4.58$) \quad p=0.012$

3.54 (1.23 to 10.16$) \quad p=0.019$

$1.21(0.65$ to 2.27$) \quad p=0.548$

1.00 (reference)

1.73 (0.94 to 3.17$) \quad p=0.080$

$3.89(1.44$ to 10.50$) \quad p=0.007$

\section{Avoid emergency hospital admissions 0-4 weeks before death (reference=No)}

Not received palliative care

0-3 weeks palliative care

$4+$ weeks palliative care

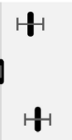

H

H
1.78 (1.39 to 2.27$) p<0.001$

1.00 (reference)

$2.04(1.60$ to 2.60$) p<0.001$

\subsection{0 \\ Odds Ratio (logarithmic scale)}

Figure 1 Multivariable adjusted ORs from logistic and multinomial logistic regression models for end-of-life outcomes by time between first contact with palliative care and death cut-off points.

increasing doses of opioids as their disease progresses. ${ }^{27}$ We found access to palliative care was associated with being two times as likely to have access to strong opioids. However, whether the referral to palliative care triggers the opioid prescription or the opioid prescription triggers the palliative care referral is unclear.
Administration of chemotherapy close to death usually represents poorly planned care. ${ }^{28}$ It was encouraging to find that only $5 \%$ of our study population received chemotherapy within the last 4 weeks of life, however, this limited the potential to explore the impact of palliative care on late chemotherapy. Studies that have established 
an association between palliative care team involvement and lower rates of chemotherapy near the end of life have concluded that cessation of chemotherapy is due to palliative care involvement. ${ }^{29}$ Although a referral to palliative care may help protect against late chemotherapy, our findings suggest this association is more complex and in some cases receiving chemotherapy or the cessation of chemotherapy may in fact trigger the palliative care referral.

We hypothesised that a deceased person who received palliative care would be more likely to avoid emergency hospital admissions in the last 4 weeks of life though we found the opposite. Further analysis revealed that only decedents in whom first contact with palliative care was longer than 4 weeks before death benefited in this outcome. Decedents whose first contact occurred less than 4 weeks before death were more likely to require emergency admission within the last 4 weeks of life. This might be explained by emergency hospital admission triggering palliative care involvement. Current evidence reports that $77 \%$ of emergency cancer admissions are avoidable,$^{30}$ so our findings indicate that there is considerable scope to reduce emergency admissions provided palliative care is initiated at least 4 weeks before death.

This study has limitations. First, the population is derived from a single UK city. Though broadly representative of the UK cancer population in prevalence of cancer type, age, sex and survival, the extent to which the level of palliative care involvement is representative of national and international activity is harder to determine. Second, the data are derived from a live clinical system and as such are likely to represent errors or omissions inherent within the system. This was moderated by restricting linkage to a single electronic system (SystmOne) which had the best potential for reliable linkage. We were unable to include data on prevalence and severity of specific symptoms and underlying disease (especially at time of any referral to palliative care) as these are not routinely coded in UK health data. It cannot be assumed therefore that referral to or longer interval between first contact with palliative care and death caused better outcomes. However, for all patients within our study population, the decision as to whether they received palliative care was made on rigorously applied eligibility criteria. ${ }^{31}$ This provides greater confidence in asserting that most if not all of the $65 \%$ of patients in our cohort who were referred to palliative care had active, progressive advanced disease and a high symptom burden, and that most if not all of the $35 \%$ of patients who did not receive palliative care had stable inactive disease. It is therefore unlikely that a lower symptom burden or disease severity among the palliative care population explains more than a very small component of our observed results.

For some patients and for some outcomes (such as access to strong opioids or cessation of chemotherapy), it is possible that the outcome event itself triggered referral to palliative care. These are nevertheless important hypotheses to test further in terms of operationalising earlier integration of palliative care. In contrast, deaths outside hospital and increased home death appear more likely to be the result of longer interval from first contact with palliative care.

\section{CONCLUSION}

The research evidence to support early integration of palliative care for patients with cancer ${ }^{1-6}$ is based on relatively high-intensity interventions of at least 8-12 weeks initiated approximately 6-14 months before death. Within routinely collected data, we have determined an association between longer interval from first contact with palliative care to death and important quality indicators of end-of-life care. Palliative care initiated more than 2 weeks before death was associated with avoiding a hospital death; and initiated more than 4 weeks before death was associated with a reduction in emergency hospital admissions and an increased likelihood of receiving an opioid analgesic. Palliative care initiated more than 32 weeks before death was associated with a reduction in chemotherapy in the last 4 weeks of life.

Characterising the impact of palliative care services based on interval between first contact and death provides new evidence which will aid policy-makers when modelling palliative care service provision. Evidence of benefit in advanced non-cancer diseases remains unclear but together with other observational evidence, our findings should stimulate similar research in these populations.

Acknowledgements The authors would like to acknowledge the key role of Professor Rick Jones who advised on the data linkage process for this study, and Chris Jackson for his role in constructing the searches and accessing the data.

Contributors Conception and design: LEZ, RMW, GH, PC, AH, PM-M, MIB. Collection and assembly of data: LEZ, CLC. Data analysis and interpretation: LEZ, CLC, RMW, MIB. Manuscript writing: all authors. Final approval of manuscript: all authors. Accountable for all aspects of the work: all authors.

Funding This study was funded by Yorkshire CancerResearch (Award L384).

Disclaimer The views expressed in this report are those of the authors and do not necessarily represent those of Yorkshire Cancer Research. The funders had no role in the study design, data collection, data analysis, data interpretation or writing of the report.

Competing interests None declared.

Ethics approval The National Research Ethics Service (PR 13.YH.0301) granted ethical approval for the study.

Provenance and peer review Not commissioned; externally peer reviewed.

Data sharing statement № additional data are available.

Open Access This is an Open Access article distributed in accordance with the Creative Commons Attribution Non Commercial (CC BY-NC 4.0) license, which permits others to distribute, remix, adapt, build upon this work non-commercially, and license their derivative works on different terms, provided the original work is properly cited and the use is non-commercial. See: http://creativecommons.org/ licenses/by-nc/4.0/

(C) Article author(s) (or their employer(s) unless otherwise stated in the text of the article) 2018. All rights reserved. No commercial use is permitted unless otherwise expressly granted. 


\section{REFERENCES}

1. Smith TJ, Temin S, Alesi ER, et al. American Society of Clinical Oncology provisional clinical opinion: the integration of palliative care into standard oncology care. J Clin Oncol 2012;30:880-7.

2. Rugno FC, Paiva BS, Paiva CE. Early integration of palliative care facilitates the discontinuation of anticancer treatment in women with advanced breast or gynecologic cancers. Gynecol Oncol 2014;135:249-54.

3. Bakitas MA, Tosteson TD, Li Z, et al. Early Versus Delayed Initiation of Concurrent Palliative Oncology Care: Patient Outcomes in the ENABLE III Randomized Controlled Trial. J Clin Oncol 2015;33:1438-45.

4. Greer JA, Jackson VA, Meier DE, et al. Early integration of palliative care services with standard oncology care for patients with advanced cancer. CA Cancer J Clin 2013;63:349-63.

5. Temel JS, Greer JA, Muzikansky A, et al. Early palliative care for patients with metastatic non-small-cell lung cancer. $N$ Engl J Med 2010;363:733-42.

6. Zimmermann C, Swami N, Krzyzanowska M, et al. Early palliative care for patients with advanced cancer: a cluster-randomised controlled trial. Lancet 2014;383:1721-30.

7. Osta BE, Palmer JL, Paraskevopoulos T, et al. Interval between first palliative care consult and death in patients diagnosed with advanced cancer at a comprehensive cancer center. J Palliat Med 2008;11:51-7.

8. Bennett MI, Ziegler L, Allsop M, et al. What determines duration of palliative care before death for patients with advanced disease? A retrospective cohort study of community and hospital palliative care provision in a large UK city. BMJ Open 2016;6:e012576.

9. Henson L, Gao W, Higginson I, et al. Emergency department attendance by patients with cancer in the last month of life: a systematic review and meta-analysis. Lancet 2015;385(Suppl 1):S41.

10. Seow H, Brazil K, Sussman J, et al. Impact of community based, specialist palliative care teams on hospitalisations and emergency department visits late in life and hospital deaths: a pooled analysis. BMJ 2014;348:g3496.

11. Blackhall LJ, Read P, Stukenborg G, et al. CARE Track for Advanced Cancer: Impact and Timing of an Outpatient Palliative Care Clinic. $J$ Palliat Med 2016;19:57-63.

12. Bharadwaj P, Helfen KM, Deleon LJ, et al. Making the Case for Palliative Care at the System Level: Outcomes Data. J Palliat Med 2016;19:255-8.

13. National Council of Palliative Care. Commissioning guidance for specialist palliative care: helping to deliver commissioning objectives, december 2012, guidance document published collaboratively with the association for palliative medicine of great Britain and Ireland, consultant nurse in palliative care reference group, Marie Curie Cancer Care. London UK: National Council for palliative Care and Palliative care Section of the Royal Society of Medicine, 2012.

14. Department of Health. End of Life Care Strategy - promoting high quality care for all adults at the end of life. London, Uk: London Central Office of Information, Crown, 2008.
15. National Institute for Health and Care Excellence. Quality standard for end of life care for adults, end of life care. London, UK: National Institute for Health and Care Excellence, 2011. (ed Issued August 2011 last modified October 2013)

16. Ziegler L, Mulvey M, Blenkinsopp A, et al. Opioid prescribing for patients with cancer in the last year of life: a longitudinal population cohort study. Pain 2016;157:2445-51.

17. Dunn OJ. Multiple comparisons using rank sums. Technometrics 1964;6:241-52.

18. McKenzie DP, McGorry PD, Wallace CS, et al. Constructing a minimal diagnostic decision tree. Methods Inf Med 1993;32:161-6.

19. National Institute of Clinical Excellence. Safe and effective prescribing of strong opioids in palliative care of adults. London, UK: National Institute of Clinical Excellence, 2011.

20. Office for National Statistics:. Statistical bulletin: national survey of bereaved people NOICES. England: ONS, 2015.

21. Gao W, Ho YK, Verne J, et al. GUIDE_Care project. Changing patterns in place of cancer death in England: a population-based study. PLoS Med 2013;10:e1001410.

22. Dixon J, King D, Matosevic T, et al. Equity in the provision of palliative care in the UK; review of Evidence PSSRU Discussion Paper 2894. London, UK: London School of Economics, 2015.

23. Luckett T, Davidson PM, Lam L, et al. Do community specialist palliative care services that provide home nursing increase rates of home death for people with life-limiting illnesses? A systematic review and meta-analysis of comparative studies. J Pain Symptom Manage 2013;45:279-97.

24. Gomes B, Calanzani N, Curiale V, et al. Effectiveness and costeffectiveness of home palliative care services for adults with advanced illness and their caregivers. Cochrane Database Syst Rev 2013:Cd007760.

25. National Institute for Health and Clinical Excellence. Safe and effective prescribing of strong opioids for pain in palliative care of adults, opioids in palliative care: NICE clinical quideline No.140. Cardiff (UK): National Collaborating Centre for Cancer, 2012.

26. Gagnon B, Scott S, Nadeau L, et al. Patterns of community-based opioid prescriptions in people dying of cancer. J Pain Symptom Manage 2015;49:36-44.

27. van den Beuken-van Everdingen MH, de Rijke JM, Kessels AG, et al. Prevalence of pain in patients with cancer: a systematic review of the past 40 years. Ann Oncol 2007;18:1437-49.

28. Pirl WF, Greer JA, Irwin K, et al. Processes of discontinuing chemotherapy for metastatic non-small-cell lung cancer at the end of life. J Oncol Pract 2015;11:e405-12.

29. Karim SM, Zekri J, Abdelghany E, et al. Time from last chemotherapy to death and its correlation with the end of life care in a referral hospital. Indian J Med Paediatr Oncol 2015;36:55-9.

30. Delgado-Guay MO, Kim YJ, Shin SH, et al. Avoidable and unavoidable visits to the emergency department among patients with advanced cancer receiving outpatient palliative care. J Pain Symptom Manage 2015;49:497-504.

31. Bennett M, Adam J, Alison D, et al. Leeds eligibility criteria for specialist palliative care services. Palliat Med 2000;14:157-8. 